\title{
Teoria da Medida e outras contribuições
}

CHAIM SAMUEL HÖNIG

$\mathrm{H}$ Á UMA DIFICULDADE NATURAL para perceber a importância de descobertas e invenções passadas e o seu impacto na nossa civilização, nos nossos conhecimentos e na nossa concepção do mundo, mesmo quando essas fazem parte do nosso cotidiano. Assim, por exemplo, dificilmente podemos imaginar como era a vida há um ou dois séculos quando não havia os meios de transporte e de comunicação atuais, nem as vacinas e a medicina moderna ... nem o micro... Nascidos e formados dentro de uma civilização nos é difícil imaginar outra sociedade completamente diferente, até em questões muito mais específicas como a situação das Ciências Matemáticas na USP e no Brasil, há 50 anos. Para quem não viveu o período correspondente, em que grandes modificações se operaram, é difícil imaginar qual era a realidade há quarenta ou sessenta anos; qual era o status da Análise, o que era ensinado como Álgebra e Geometria etc.; qual o número de estudantes, o seu nível e as suas condições de estudo, a estrutura das Universidades, as condições de carreira etc. Do mesmo modo, para matemáticos das duas últimas gerações, é muito difícil conceber o impacto e a originalidade das contribuições de von Neumann pois nós já nascemos dentro das mudanças provocadas por elas.

Uma das características de von Neumann era a simplicidade que ele conseguia ver em situações aparentemente muito complicadas. Ao mesmo tempo, ele também sabia trabalhar com áreas extremamente ricas e complexas, dando-lhes forma, organização e estrutura que permaneceriam por décadas. Nas exposições de hoje veremos diversos exemplos disso.

Além disso, para avaliarmos uma contribuição científica devemos começar por colocá-la no seu contexto, tanto com relação ao passado, aos problemas que ela resolveu, quanto com relação ao futuro e às novas direções que sugeriu ou sugere e estimula. Porém, acontece às vezes que o passado nada diz, pois se trata de problema que nãa existia ou de que ninguém sequer cogitava de uma possível solução. Isto aconteceu freqüentemente nas contribuições de von Neumann. 
Nesta exposição nos deteremos em particular nas contribuições de von Neumann à Teoria da Medida e a alguns tópicos da Análise. Suas contribuições mais importantes e difíceis a Álgebras de Operadores e a outras áreas serão abordadas pelos conferencistas que me seguirão.

Uma das criações mais importantes da Análise do começo deste século foi a da Integral de Lebesgue. Até então o grande instrumento para medida de grandezas era a Integral de Riemann. A Integral de Lebesgue (cujas propriedades e condições de aplicação são muito simples) não só estendeu notavelmente a Integral de Riemann como permitiu resolver, num período de poucos anos, os problemas fundamentais da Teoria da Integração que estavam em aberto: problemas de séries de Fourier (o Teorema de Fischer-Riesz), teoremas de convergência de integrais, condições da iteração de integrais (o Teorema de Fubini) etc., além de dar um impulso decisivo à Análise Funcional, às Teorias das Equações Diferenciais e Integrais etc. (pois com a Integral de Lebesgue passamos a ter espaços completos). $\mathrm{O}$ ponto básico dessa nova teoria foi a introdução da noção de medida, estendendo as clássicas noções de comprimento, área, volume etc. Desenvolveu-se então a Teoria da Medida e a Teoria das Funções Mensuráveis. No livro de Lebesgue e nos de outros autores das décadas seguintes esta parte preliminar da construção da medida (a definição de conjunto mensurável através da coincidência da medida interior e da medida exterior do conjunto) é muito trabalhosa e extensa. Mesmo no livro de Titchmarch (1a. ediçâo 1932), só a parte da Teoria da Medida ocupava quinze páginas; a definição simplificada que Caratheodory deu de conjunto mensurável melhorou a situação. (Do ponto de vista da Análise Funcional houve diversas extensões da Integral de Lebesgue, em particular a formulação de Daniell.) Restava porém simplificar a demonstração de que a nova teoria estendia as noções clássicas; por exemplo, que com a nova definição de medida valia o fato evidente de que a medida de um retângulo coincidia com o produto do comprimento de seus lados.

A demonstração geométrica deste fato é elementar mas extremamente longa e, por esta razão, não é feita nos textos. Von Neumann deu uma demonstração muito engenhosa e simples deste fato: ele recobriu o retângulo por um conjunto (infinito) de retângulos, conjunto este que por compacidade sempre pode ser tomado finito; a seguir demonstrou, por contagem de pontos de um reticulado e por homotetia, que a soma das áreas dos retângulos recobridores é sempre maior que a do retângulo inicial. Essa demonstração (tirada das notas de aula de um curso de von Neumann) é reproduzida no livro Differential Topology de Guillemin e Pollack e ocupa menos de uma página (p. 203). 

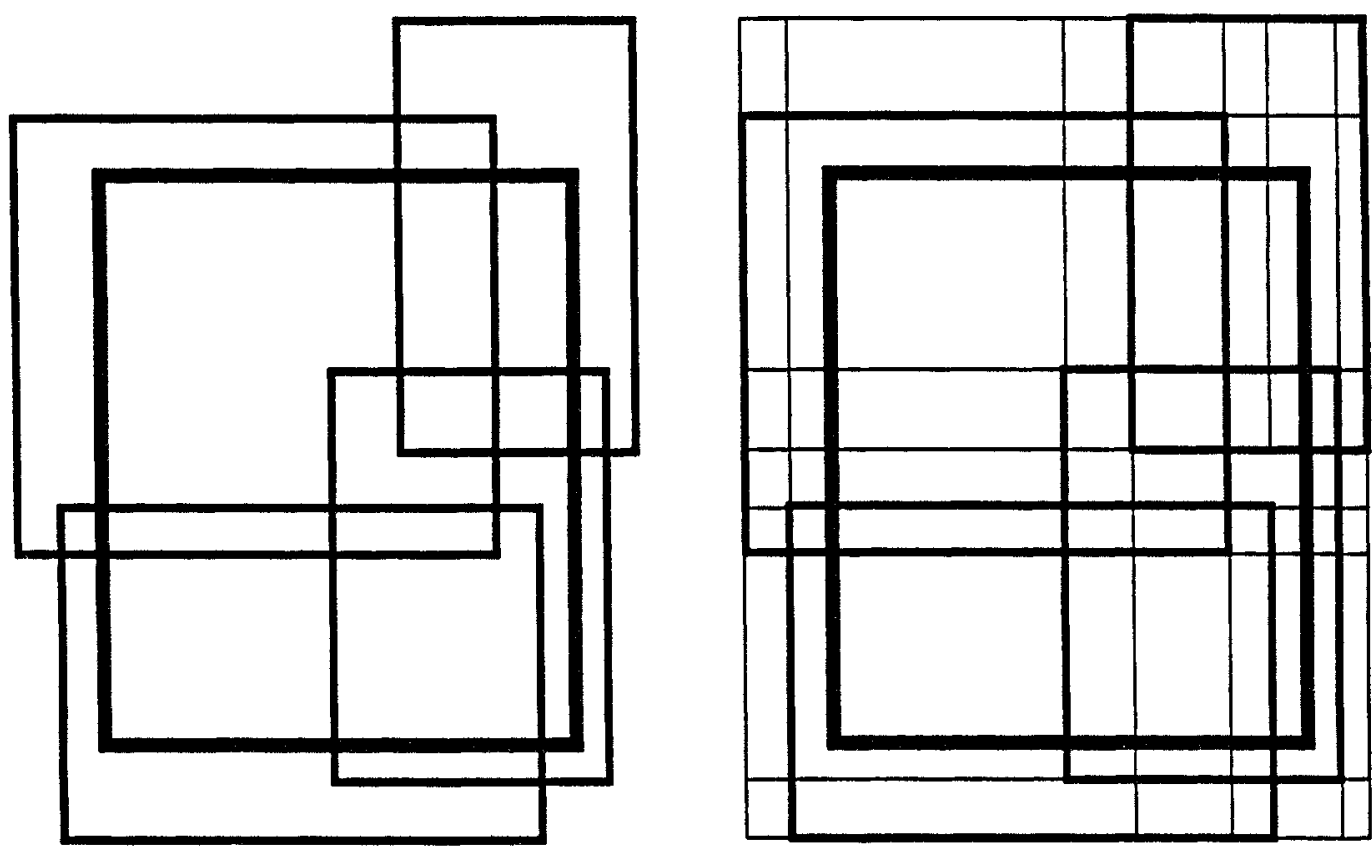

Durante a Segunda Guerra Mundial, quando von Neuman trabalhou no projeto Manhattan para a construção da bomba atômica, usou uma idéia análoga para calcular a configuração geométrica adequada à bomba. Mencionemos que a demonstração elementar acima de von Neumann (ou a definição direta da integral feita posteriormente, independentemente, por Kurzweil, Henstock \& Mc Shane) permite que hoje a Teoria de Integração de Lebesgue seja desenvolvida muito rapidamente.

Mencionemos ainda que outra contribuição relevante de Von Neumann na Teoria da Medida é a demonstração da existência e unicidade da Medida de Haar em grupos compactos. Cumpre também lembrar a. extensão que von Neumann fez do paradoxo de Banach-Tarski a grupos não abelianos e suas contribuições ao quinto problema de Hilbert para. grupos compactos.

Von Neumann ainda tem contribuições a muitas outras áreas como Lógica, Teoria dos Conjuntos, Álgebras e Reticulados (particularmente foi um dos criadores das Álgebras de Jordan (Jordan et al., 1934)), Fundamentos, Teoria Ergódica (deu o primeiro tratamento rigoroso da hipótese ergódica em mecânica estatística), Hidrodinâmica etc., bem como o seu papel primordial na Computação, Economia Matemática, Teoria dos Jogos e Mecânica Quântica, sobre as quais versarão as outras exposições de hoje. 
Proposition. Let $S$ be a rectangular solid and $S_{1}, S_{2}, \ldots$ a covering of its closure of $\bar{S}$ by other solids. Then $\sum \operatorname{vol}\left(S_{j}\right) \geq \operatorname{vol}(S)$.

Proof. Call $\left(z_{1}, \ldots, z_{n}\right)$ an integer point of $\mathbf{R}^{n}$ if each $z_{1}$ is an integer. Let $S=S(a, b)$. The number of integers in the interval $a_{i}<z_{i}<b_{i}$ is less than $b_{i}-a_{i}+1$ and at least as large as $b_{t}-a_{i}-1$. Assume, for the moment, that the length $b_{i}-a_{i}$ of each side of $S$ is greater than 1 . Then the number of integer points in $S$ is less than

$$
\prod_{i=1}^{n}\left(b_{i}-a_{i}+1\right)
$$

and at least

$$
\prod_{i=1}^{n}\left(b_{i}-a_{i}-1\right)
$$

If $S_{1}, S_{2}, \ldots$ is a cover of $\bar{S}$, then, by compactness, some finite number $S_{1}, \ldots, S_{N}$ also is a cover. Now the number of integer points in $S$ is at most equal to the sum of the numbers of integer points in $S_{1}, \ldots, S_{N}$. Thus if $S_{j}=S\left(a^{\prime}, b^{\prime}\right)$, we get

$$
\prod_{i=1}^{n}\left(b_{i}-a_{i}-1\right) \leq \sum_{j=1}^{N} \prod_{i=1}^{n}\left(b_{i}^{\prime}-a_{i}^{\prime}+1\right) .
$$

For each positive number $\lambda$, define $\lambda S(a, b)=S(\lambda a, \lambda b)$. Since $\overline{\lambda S}$ is covered by $\lambda S_{1}, \ldots, \lambda S_{N}$ the preceding calculation gives

$$
\prod_{i=1}^{n}\left(\lambda b_{i}-\lambda a_{i}-1\right) \leq \sum_{j=1}^{N} \prod_{i=1}^{n}\left(\lambda b_{i}^{j}-\lambda a_{i}^{j}+1\right) .
$$

Now, no matter what the size of $S$, if $\lambda$ is large enough, then $\lambda S$ will have sides of length greater than 1 . So the last inequality is valid for large $\lambda$ without any restriction on $S$.

You have now only to divide both sides of the inequality by $\lambda^{n}$ and then let $\lambda \rightarrow \infty$ to complete the proof. Q.E.D.

É também notável o legado de von Neumann quando analisado sob a perspectiva da descendência a que deu lugar. Lembremos o padrão criado em meados do século passado pela teoria das Superfícies de Riemann, a qual culmina com a fórmula de Riemann-Roch que liga completamente os aspectos analíticos, geométricos e algébricos do estudo dessas superfícies. Este estudo serviu de modelo para teorias posteriores; assim, por exemplo, a teoria de Atiyah-Singer de equações a derivadas parciais em variedades. Analogamente, o estudo das Álgebras de von Neumann levou diretamente à Matemática não-comutátiva (Geometria nãocomutativa (Connes, 1994), Integração não-comutativa, Teoria das Pro- 
babilidades não-comutativa, Teoria dos Conjuntos não-comutativa etc. o nome não-comutativo se deve ao fato de que a Álgebra correspondente às estruturas precedentes é não-comutativa). Esta linha, que hoje é uma das mais ativas da matemática, teve em particular dois matemáticos (Allain Connes, 1978 e Vaughan Jones, 1990) contemplados com a medalha Field, (o equivalente, em Matemática, ao prêmio Nobel), outorgada a cada quatro anos pelo Congresso Internacional de Matemática.

Referências bibliográficas

CONNES, A. Non-commutative geometry, Academic Press, 2 ed., 1994.

JORDAN, P.; VON NEUMANN, J. \& WIGNER, E. On the algebraic generalization of the quantum mechanical formalism. Annals of Mathematics, v. 35, p. $29-64,1934$.

Chaim Samuel Hönig é professor do Departamento de Matemática do Instituto de Matemática e Estatística da USP.

Palestra feita pelo autor no encontro A obra e o legado de John von Neumann, organizado pelo Instituto de Estudos Avançados da USP e pela Academia Brasileira de Ciências no Instituto de Matemática e Estatística da USP em 14 de novembro de 1995. 